\title{
Tax rates effects on the risk level of listed Viet Nam real estate firms during global economic crisis 2007-2009
}

\section{Dinh Tran Ngoc Huy}

Banking University, HCMC - GSIM, International University of Japan, Japan

$M B A, P h D$ candidate

\section{open $(2$ access (c) dol}

Article history:

Received: March 25, 2018

1st Revision: May 9, 2018

Accepted: May 20, 2018

\section{JEL classification:}

G01

G10

G39

DOI:

10.14254/jems.2018.3-2.3

\begin{abstract}
The emerging stock market in Viet Nam has been developed since 2006 and affected by the financial crisis 2007-2009. This study analyzes the impacts of tax policy on market risk for the listed firms in the real estate industry as it becomes necessary. First, by using quantitative and analytical methods to estimate asset and equity beta of total 45 listed companies in Viet Nam real estate industry with a proper traditional model, we found out that the beta values, in general, for many institutions are acceptable. Second, under 3 different scenarios of changing tax rates $(20 \%, 25 \%$ and $28 \%)$, we recognized that there is not large disperse in equity beta values, estimated at $0.750,0.762$ and 0.769 . Third, by changing tax rates in 3 scenarios $(25 \%, 20 \%$ and $28 \%$ ), we recognized both equity and asset beta mean values have positive relationship with the increasing levels of tax rate. Finally, this paper provides some outcomes that could provide companies and government more evidence in establishing their policies in governance.
\end{abstract}

Keywords: beta, capital structure, economic crisis, risk, tax rate, real estate industry.

\section{Introduction}

Together with the development of the whole economy and the growth of FDI, throughout many recent years, Viet Nam real estate industry is considered as one of active economic sectors, which has some positive effects for the economy.

This paper is organized as follow. The research issues and literature review will be covered in next sessions 2 and 3, for a short summary. Then, methodology and conceptual theories are introduced in session 4 and 5. Session 6 describes the data in empirical analysis. Session 7 presents empirical results and findings. Next, session 8 covers the analytical results. Then, session 9 presents analysis of risk. Lastly, session 10 will conclude with some policy suggestions. This paper also supports readers with references, exhibits and relevant web sources. 


\section{Research Issues}

We mention some issues on the estimating of impacts of tax rates on beta for listed real estate companies in Viet Nam stock exchange as following:

Issue 1: Whether the risk level of real estate firms under the different changing scenarios of tax rates increase or decrease so much.

Issue 2: Whether the disperse distribution of beta values become large in the different changing scenarios of tax rates estimated in the real estate industry.

Beside, we also propose some hypotheses for the above issues:

Hypothesis 1: because tax may strongly affect business returns, changing tax scenarios could strongly affect firm risk.

Hypothesis 2: as tax policy is vital for the business development, there will be large disperse in beta or risk values estimated.

\section{Literature review}

Smith (2004) mentions in Chicago, properties located in a designated TIF (tax increment financing) district will exhibit higher rates of appreciation after the area is designated a qualifying TIF district when compared to those properties selling outside TIF districts, and when compared to properties that sell within TIF district boundaries prior to designation.

David (2009) stated the U.S states can increase the likelihood of using tax rate adjustments to cope with fiscal volatility rather than (more harmful) spending fluctuations. Robert et all (2011) recognized a significant positive relation between changes in intercorporate investment and changes in corporate marginal tax rates on ordinary income.

George and Jot Yau (2012) found that there is a positive relationship between transaction cost and price volatility, suggesting that the imposition of a transaction tax could increase financial market fragility, increasing the likelihood of a financial crisis rather than reducing it. Mark (2012) found in some European countries during the crisis raising taxe rates and tax burdens, the trend in which overall revenue levels were broadly stable while marginal rates in corporate and top personal income declined has stopped. Then, Filip (2012) believed low levels of taxation, esp. low levels of taxation on the income or wealth of the so-called productive segments of society are beneficial for economic growth.

Finally, tax rate can be considered as one among many factors that affect business risk of real estate firms.

\section{Conceptual theories}

\section{The impact of fiscal policy on the economy}

Tax policy is one among major fiscal policies. When the government decides to change the tax policy or tax rates, the mobility of capital in the markets will be affected.

In a specific industry such as real estate industry, on the one hand, using tax policy with a decrease or increase in tax rate could affect tax revenues, profit after tax and financial results and compensation and jobs of the industry. And it also shows the purpose of fiscal policy: following either contractionary or expansionary directions.

During and after financial crises such as the 2007-2009 crisis, there raises concerns about fiscal policies or public policies of many countries, in both developed and developing markets. The government might choose either lowering the tax rates or cutting the public expenditures while increasing demand stimulating programs to resolve difficulties from the crisis.

\section{Methodology}

In this study, we use the live data during the crisis period 2007-2011 from the stock exchange market in Viet Nam (HOSE and HNX) to estimate systemic risk results and tax impacts.

In this research, analytical research method is used, philosophical method is used and specially, tax rate scenario analysis method is used. Analytical data is from the situation of listed insurance firms in VN stock exchange and current tax rate is $25 \%$.

Finally, we use the results to suggest policy for both these enterprises, relevant organizations and government. 


\section{General Data Analysis}

The research sample has total 45 listed firms in the real estate market with the live data from the stock exchange.

Firstly, we estimate equity beta values of these firms and use financial leverage to estimate asset beta values of them. Secondly, we change the tax rate from $25 \%$ to $28 \%$ and $20 \%$ to see the sensitivity of beta values. We found out that in 3 cases (rate $=20 \%, 25 \%$, and 28\%), asset beta mean is estimated at $0.362,0.367$ and 0.370 which are negatively correlated with tax rate. Also in 3 scenarios, we find out var of asset beta estimated at 0.750, 0.762 and 0.769 (almost the same) which shows acceptable risk dispersion. Tax rate changes almost has no effect on asset beta var under financial leverage.

\section{Empirical Research Findings and Discussion}

In the below section, data used are from total 45 listed insurance companies on VN stock exchange (HOSE and HNX mainly). In the scenario 1, current tax rate is $25 \%$ which is used to calculate market risk (beta). Then, two (2) tax rate scenarios are changed up to $28 \%$ and down to $20 \%$, compared to the current corporate tax rate.

Market risk (beta) under the impact of tax rate, includes: 1) equity beta; and 2) asset beta.

\subsection{Scenario 1: Current tax rate is $25 \%$}

In the case of tax rate of $25 \%$, all beta values of 45 listed firms on VN real estate market as following:

\begin{tabular}{|c|c|c|c|c|c|}
\hline Order No. & $\begin{array}{c}\text { Company stock } \\
\text { code }\end{array}$ & Equity beta & $\begin{array}{c}\text { Asset beta } \\
\text { (assume debt } \\
\text { beta = 0) }\end{array}$ & Note & $\begin{array}{l}\text { Financial } \\
\text { leverage }\end{array}$ \\
\hline 1 & API & 1.686 & 1.580 & $\begin{array}{c}\text { RCL as } \\
\text { comparable }\end{array}$ & $6.3 \%$ \\
\hline 2 & ASM & 0.495 & 0.173 & $\begin{array}{c}\text { HDC as } \\
\text { comparable }\end{array}$ & $65.0 \%$ \\
\hline 3 & $\mathrm{BCI}$ & 1.161 & 0.523 & & $55.0 \%$ \\
\hline 4 & CCI & 0.476 & 0.145 & $\begin{array}{c}\text { UIC as } \\
\text { comparable }\end{array}$ & $69.4 \%$ \\
\hline 5 & CLG & 0.383 & 0.092 & $\begin{array}{c}\text { UIC as } \\
\text { comparable }\end{array}$ & $75.9 \%$ \\
\hline 6 & D2D & 1.446 & 0.533 & & $63.2 \%$ \\
\hline 8 & DLG & 0.596 & 0.198 & $\begin{array}{c}\text { SC5 as } \\
\text { comparable }\end{array}$ & $66.9 \%$ \\
\hline 9 & DTA & 0.974 & 0.466 & $\begin{array}{c}\text { RCL as } \\
\text { comparable }\end{array}$ & $52.2 \%$ \\
\hline 10 & DXG & 0.145 & 0.046 & $\begin{array}{c}\text { LGL as } \\
\text { comparable }\end{array}$ & $68.4 \%$ \\
\hline 11 & HAG & 0.632 & 0.295 & & $53.3 \%$ \\
\hline 12 & HDC & 1.185 & 0.425 & & $64.2 \%$ \\
\hline 13 & HDG & 0.253 & 0.099 & $\begin{array}{c}\text { LHG as } \\
\text { comparable }\end{array}$ & $61.0 \%$ \\
\hline 14 & IDJ & 1.198 & 0.776 & $\begin{array}{c}\text { API as } \\
\text { comparable }\end{array}$ & $35.2 \%$ \\
\hline 15 & IDV & 0.428 & 0.082 & $\begin{array}{c}\text { RCL as } \\
\text { comparable }\end{array}$ & $80.7 \%$ \\
\hline 16 & IJC & 0.411 & 0.120 & $\begin{array}{c}\mathrm{BCI} \text { as } \\
\text { comparable }\end{array}$ & $70.9 \%$ \\
\hline 17 & ITA & 1.121 & 0.749 & & $33.2 \%$ \\
\hline 18 & ITC & 0.591 & 0.338 & $\begin{array}{c}\text { NBB as } \\
\text { comparable }\end{array}$ & $42.8 \%$ \\
\hline 19 & $\mathrm{KBC}$ & 0.945 & 0.371 & & $60.7 \%$ \\
\hline 20 & $\mathrm{KDH}$ & 1.071 & 0.670 & $\begin{array}{c}\text { LCG as } \\
\text { comparable }\end{array}$ & $37.5 \%$ \\
\hline 21 & LCG & 1.552 & 0.923 & & $40.5 \%$ \\
\hline 22 & LGL & 0.381 & 0.168 & $\begin{array}{c}\text { PPI as } \\
\text { comparable }\end{array}$ & $56.1 \%$ \\
\hline 23 & LHG & 0.548 & 0.215 & $\begin{array}{c}\text { DLG as } \\
\text { comparable }\end{array}$ & $60.8 \%$ \\
\hline
\end{tabular}




\begin{tabular}{|c|c|c|c|c|c|}
\hline 24 & NBB & 0.923 & 0.317 & & $65.6 \%$ \\
\hline 25 & NHA & 1.399 & 1.034 & $\begin{array}{c}\text { RCL as } \\
\text { comparable }\end{array}$ & $26.1 \%$ \\
\hline 26 & NTL & 1.557 & 0.701 & & $55.0 \%$ \\
\hline 27 & NVN & 0.167 & 0.061 & $\begin{array}{c}\text { CLG as } \\
\text { comparable }\end{array}$ & $63.3 \%$ \\
\hline 28 & OGC & 0.593 & 0.271 & $\begin{array}{l}\text { ITA as } \\
\text { comparable }\end{array}$ & $54.3 \%$ \\
\hline 29 & PDR & 0.194 & 0.078 & IJC as comparable & $59.9 \%$ \\
\hline 30 & PPI & 0.746 & 0.332 & $\begin{array}{c}\text { D2D as } \\
\text { comparable }\end{array}$ & $55.5 \%$ \\
\hline 31 & PVL & 0.110 & 0.078 & $\begin{array}{c}\text { DXG as } \\
\text { comparable }\end{array}$ & $29.6 \%$ \\
\hline 32 & QCG & 0.718 & 0.290 & SJS as comparable & $59.5 \%$ \\
\hline 33 & RCL & 1.770 & 0.991 & & $44.0 \%$ \\
\hline 34 & SC5 & 1.497 & 0.240 & & $84.0 \%$ \\
\hline 35 & SDU & 0.128 & 0.053 & $\begin{array}{c}\text { VCR as } \\
\text { comparable }\end{array}$ & $58.5 \%$ \\
\hline 36 & SJS & 1.509 & 0.799 & & $47.1 \%$ \\
\hline 37 & SZL & 0.425 & 0.258 & & $39.3 \%$ \\
\hline 38 & TDH & 1.103 & 0.722 & & $34.5 \%$ \\
\hline 39 & TIX & 0.202 & 0.082 & $\begin{array}{c}\text { SZL as } \\
\text { comparable }\end{array}$ & $59.6 \%$ \\
\hline 40 & UDC & 0.216 & 0.071 & $\begin{array}{c}\text { LHG as } \\
\text { comparable }\end{array}$ & $67.2 \%$ \\
\hline 41 & UIC & 1.286 & 0.357 & & $72.2 \%$ \\
\hline 42 & VCR & 0.263 & 0.165 & $\begin{array}{c}\text { LGL as } \\
\text { comparable }\end{array}$ & $37.4 \%$ \\
\hline 43 & VIC & 0.755 & 0.186 & & $75.4 \%$ \\
\hline 44 & VPH & 0.070 & 0.019 & $\begin{array}{c}\text { UDC as } \\
\text { comparable }\end{array}$ & $73.5 \%$ \\
\hline 45 & VRC & 0.203 & 0.073 & $\begin{array}{c}\text { CCI as } \\
\text { comparable }\end{array}$ & $64.1 \%$ \\
\hline
\end{tabular}

\subsection{Scenario 2: Tax rate increases up to $28 \%$}

If corporate tax rates increases up to $28 \%$, all beta values of total 45 listed firms on VN real estate market as below:

\begin{tabular}{|c|c|c|c|c|c|}
\hline Order No. & $\begin{array}{c}\text { Company stock } \\
\text { code }\end{array}$ & Equity beta & $\begin{array}{c}\text { Asset beta } \\
\text { (assume debt } \\
\text { beta }=0 \text { ) }\end{array}$ & Note & $\begin{array}{l}\text { Financial } \\
\text { leverage }\end{array}$ \\
\hline 1 & API & 1.6891 & 1.5835 & $\begin{array}{c}\text { RCL as } \\
\text { comparable }\end{array}$ & $6.3 \%$ \\
\hline 2 & ASM & 0.5066 & 0.1772 & $\begin{array}{l}\text { HDC as } \\
\text { comparable }\end{array}$ & $65.0 \%$ \\
\hline 3 & $\mathrm{BCI}$ & 1.1606 & 0.5225 & & $55.0 \%$ \\
\hline 4 & CCI & 0.4880 & 0.1491 & $\begin{array}{c}\text { UIC as } \\
\text { comparable }\end{array}$ & $69.4 \%$ \\
\hline 5 & CLG & 0.3938 & 0.0950 & $\begin{array}{c}\text { UIC as } \\
\text { comparable }\end{array}$ & $75.9 \%$ \\
\hline 6 & D2D & 1.4459 & 0.5326 & & $63.2 \%$ \\
\hline 8 & DLG & 0.6107 & 0.2024 & $\begin{array}{c}\text { SC5 as } \\
\text { comparable }\end{array}$ & $66.9 \%$ \\
\hline 9 & DTA & 0.9915 & 0.4742 & $\begin{array}{c}\text { RCL as } \\
\text { comparable }\end{array}$ & $52.2 \%$ \\
\hline 10 & DXG & 0.1548 & 0.0489 & $\begin{array}{l}\text { LGL as } \\
\text { comparable }\end{array}$ & $68.4 \%$ \\
\hline 11 & HAG & 0.6324 & 0.2955 & & $53.3 \%$ \\
\hline 12 & HDC & 1.1847 & 0.4247 & & $64.2 \%$ \\
\hline 13 & HDG & 0.2637 & 0.1030 & $\begin{array}{l}\text { LHG as } \\
\text { comparable }\end{array}$ & $61.0 \%$ \\
\hline 14 & IDJ & 1.2141 & 0.7866 & $\begin{array}{c}\text { API as } \\
\text { comparable }\end{array}$ & $35.2 \%$ \\
\hline 15 & IDV & 0.4409 & 0.0850 & $\begin{array}{c}\text { RCL as } \\
\text { comparable }\end{array}$ & $80.7 \%$ \\
\hline
\end{tabular}




\begin{tabular}{|c|c|c|c|c|c|}
\hline 16 & IJC & 0.4218 & 0.1229 & $\begin{array}{c}\text { BCI as } \\
\text { comparable }\end{array}$ & $70.9 \%$ \\
\hline 17 & ITA & 1.1212 & 0.7488 & & $33.2 \%$ \\
\hline 18 & ITC & 0.5999 & 0.3432 & $\begin{array}{c}\text { NBB as } \\
\text { comparable }\end{array}$ & $42.8 \%$ \\
\hline 19 & $\mathrm{KBC}$ & 0.9455 & 0.3713 & & $60.7 \%$ \\
\hline 20 & KDH & 1.0841 & 0.6780 & $\begin{array}{c}\text { LCG as } \\
\text { comparable }\end{array}$ & $37.5 \%$ \\
\hline 21 & LCG & 1.5516 & 0.9225 & & $40.5 \%$ \\
\hline 22 & LGL & 0.3967 & 0.1743 & $\begin{array}{c}\text { PPI as } \\
\text { comparable }\end{array}$ & $56.1 \%$ \\
\hline 23 & LHG & 0.5602 & 0.2199 & $\begin{array}{c}\text { DLG as } \\
\text { comparable }\end{array}$ & $60.8 \%$ \\
\hline 24 & NBB & 0.9232 & 0.3171 & & $65.6 \%$ \\
\hline 25 & NHA & 1.4110 & 1.0424 & $\begin{array}{c}\text { RCL as } \\
\text { comparable }\end{array}$ & $26.1 \%$ \\
\hline 26 & NTL & 1.5570 & 0.7011 & & $55.0 \%$ \\
\hline 27 & NVN & 0.1756 & 0.0644 & $\begin{array}{c}\text { CLG as } \\
\text { comparable }\end{array}$ & $63.3 \%$ \\
\hline 28 & OGC & 0.6041 & 0.2760 & $\begin{array}{c}\text { ITA as } \\
\text { comparable }\end{array}$ & $54.3 \%$ \\
\hline 29 & PDR & 0.2032 & 0.0815 & IJC as comparable & $59.9 \%$ \\
\hline 30 & PPI & 0.7611 & 0.3383 & $\begin{array}{c}\text { D2D as } \\
\text { comparable }\end{array}$ & $55.5 \%$ \\
\hline 31 & PVL & 0.1188 & 0.0836 & $\begin{array}{c}\text { DXG as } \\
\text { comparable }\end{array}$ & $29.6 \%$ \\
\hline 32 & QCG & 0.7331 & 0.2967 & SJS as comparable & $59.5 \%$ \\
\hline 33 & RCL & 1.7702 & 0.9910 & & $44.0 \%$ \\
\hline 34 & SC5 & 1.4974 & 0.2396 & & $84.0 \%$ \\
\hline 35 & SDU & 0.1377 & 0.0571 & $\begin{array}{c}\text { VCR as } \\
\text { comparable }\end{array}$ & $58.5 \%$ \\
\hline 36 & SJS & 1.5094 & 0.7989 & & $47.1 \%$ \\
\hline 37 & SZL & 0.4252 & 0.2579 & & $39.3 \%$ \\
\hline 38 & TDH & 1.1026 & 0.7220 & & $34.5 \%$ \\
\hline 39 & TIX & 0.2062 & 0.0833 & $\begin{array}{c}\text { SZL as } \\
\text { comparable }\end{array}$ & $59.6 \%$ \\
\hline 40 & UDC & 0.2261 & 0.0741 & $\begin{array}{l}\text { LHG as } \\
\text { comparable }\end{array}$ & $67.2 \%$ \\
\hline 41 & UIC & 1.2863 & 0.3573 & & $72.2 \%$ \\
\hline 42 & VCR & 0.2775 & 0.1738 & $\begin{array}{c}\text { LGL as } \\
\text { comparable }\end{array}$ & $37.4 \%$ \\
\hline 43 & VIC & 0.7551 & 0.1860 & & $75.4 \%$ \\
\hline 44 & VPH & 0.0753 & 0.0199 & $\begin{array}{c}\text { UDC as } \\
\text { comparable }\end{array}$ & $73.5 \%$ \\
\hline 45 & VRC & 0.2133 & 0.0765 & $\begin{array}{c}\text { CCI as } \\
\text { comparable }\end{array}$ & $64.1 \%$ \\
\hline
\end{tabular}

\subsection{Scenario 3: Tax rate decreases down to $20 \%$}

If corporate tax rate decreases down to $20 \%$, all beta values of total 45 listed firms on the real estate market in $\mathrm{VN}$ as following:

\section{Table 3: Market risk of listed real estate firms $(t=20 \%)$}

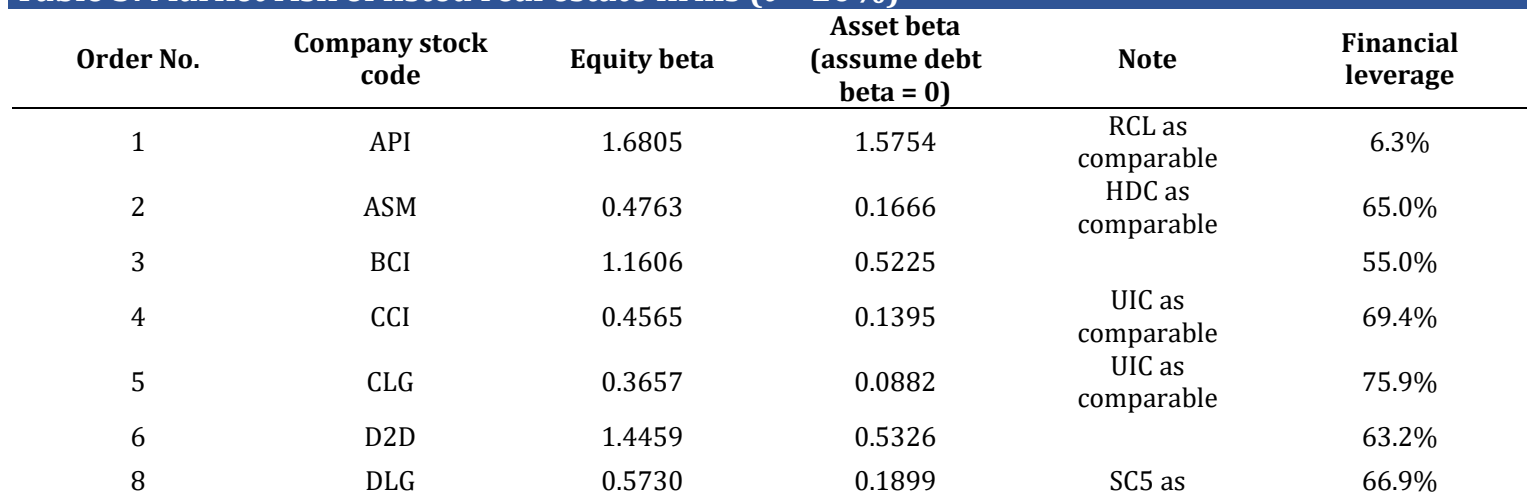




\begin{tabular}{|c|c|c|c|c|c|}
\hline & & & \multicolumn{3}{|c|}{ comparable } \\
\hline 9 & DTA & 0.9453 & 0.4521 & $\begin{array}{c}\text { RCL as } \\
\text { comparable }\end{array}$ & $52.2 \%$ \\
\hline 10 & DXG & 0.1308 & 0.0413 & $\begin{array}{c}\text { LGL as } \\
\text { comparable }\end{array}$ & $68.4 \%$ \\
\hline 11 & $\mathrm{HAG}$ & 0.6324 & 0.2955 & & $53.3 \%$ \\
\hline 12 & HDC & 1.1847 & 0.4247 & & $64.2 \%$ \\
\hline 13 & HDG & 0.2353 & 0.0919 & $\begin{array}{c}\text { LHG as } \\
\text { comparable }\end{array}$ & $61.0 \%$ \\
\hline 14 & IDJ & 1.1713 & 0.7589 & $\begin{array}{c}\text { API as } \\
\text { comparable }\end{array}$ & $35.2 \%$ \\
\hline 15 & IDV & 0.4069 & 0.0784 & $\begin{array}{c}\text { RCL as } \\
\text { comparable }\end{array}$ & $80.7 \%$ \\
\hline 16 & IJC & 0.3940 & 0.1148 & $\begin{array}{c}\text { BCI as } \\
\text { comparable }\end{array}$ & $70.9 \%$ \\
\hline 17 & ITA & 1.1212 & 0.7488 & & $33.2 \%$ \\
\hline 18 & ITC & 0.5775 & 0.3303 & $\begin{array}{c}\text { NBB as } \\
\text { comparable }\end{array}$ & $42.8 \%$ \\
\hline 19 & $\mathrm{KBC}$ & 0.9455 & 0.3713 & & $60.7 \%$ \\
\hline 20 & $\mathrm{KDH}$ & 1.0490 & 0.6560 & $\begin{array}{c}\text { LCG as } \\
\text { comparable }\end{array}$ & $37.5 \%$ \\
\hline 21 & LCG & 1.5516 & 0.9225 & & $40.5 \%$ \\
\hline 22 & LGL & 0.3578 & 0.1572 & $\begin{array}{c}\text { PPI as } \\
\text { comparable }\end{array}$ & $56.1 \%$ \\
\hline 23 & LHG & 0.5292 & 0.2077 & $\begin{array}{c}\text { DLG as } \\
\text { comparable }\end{array}$ & $60.8 \%$ \\
\hline 24 & NBB & 0.9232 & 0.3171 & & $65.6 \%$ \\
\hline 25 & NHA & 1.3799 & 1.0194 & $\begin{array}{c}\text { RCL as } \\
\text { comparable }\end{array}$ & $26.1 \%$ \\
\hline 26 & NTL & 1.5570 & 0.7011 & & $55.0 \%$ \\
\hline 27 & NVN & 0.1535 & 0.0563 & $\begin{array}{c}\text { CLG as } \\
\text { comparable }\end{array}$ & $63.3 \%$ \\
\hline 28 & OGC & 0.5746 & 0.2625 & $\begin{array}{c}\text { ITA as } \\
\text { comparable }\end{array}$ & $54.3 \%$ \\
\hline 29 & PDR & 0.1795 & 0.0719 & IJC as comparable & $59.9 \%$ \\
\hline 30 & PPI & 0.7230 & 0.3214 & $\begin{array}{c}\text { D2D as } \\
\text { comparable }\end{array}$ & $55.5 \%$ \\
\hline 31 & PVL & 0.0978 & 0.0688 & $\begin{array}{c}\text { DXG as } \\
\text { comparable }\end{array}$ & $29.6 \%$ \\
\hline 32 & QCG & 0.6934 & 0.2806 & SJS as comparable & $59.5 \%$ \\
\hline 33 & RCL & 1.7702 & 0.9910 & & $44.0 \%$ \\
\hline 34 & SC5 & 1.4974 & 0.2396 & & $84.0 \%$ \\
\hline 35 & SDU & 0.1138 & 0.0472 & $\begin{array}{c}\text { VCR as } \\
\text { comparable }\end{array}$ & $58.5 \%$ \\
\hline 36 & SJS & 1.5094 & 0.7989 & & $47.1 \%$ \\
\hline 37 & SZL & 0.4252 & 0.2579 & & $39.3 \%$ \\
\hline 38 & $\mathrm{TDH}$ & 1.1026 & 0.7220 & & $34.5 \%$ \\
\hline 39 & TIX & 0.1950 & 0.0788 & $\begin{array}{c}\text { SZL as } \\
\text { comparable }\end{array}$ & $59.6 \%$ \\
\hline 40 & UDC & 0.2003 & 0.0656 & $\begin{array}{c}\text { LHG as } \\
\text { comparable }\end{array}$ & $67.2 \%$ \\
\hline 41 & UIC & 1.2863 & 0.3573 & & $72.2 \%$ \\
\hline 42 & VCR & 0.2422 & 0.1517 & $\begin{array}{c}\text { LGL as } \\
\text { comparable }\end{array}$ & $37.4 \%$ \\
\hline 43 & VIC & 0.7551 & 0.1860 & & $75.4 \%$ \\
\hline 44 & VPH & 0.0621 & 0.0164 & $\begin{array}{c}\text { UDC as } \\
\text { comparable }\end{array}$ & $73.5 \%$ \\
\hline 45 & VRC & 0.1878 & 0.0673 & $\begin{array}{c}\text { CCI as } \\
\text { comparable }\end{array}$ & $64.1 \%$ \\
\hline
\end{tabular}

All three above tables and data show that values of equity and asset beta in the case of increasing tax rate up to $28 \%$ or decreasing rate down to $20 \%$ have small fluctuation. 


\section{Comparing statistical results in 3 scenarios of changing tax rate:}

\begin{tabular}{cccc}
$\begin{array}{c}\text { Table 4: Statistical results (tax rate } \mathbf{=} \mathbf{2 5} \%) \\
\text { Statistic results }\end{array}$ & Equity beta & $\begin{array}{c}\text { Asset beta (assume debt } \\
\text { beta } \mathbf{0})\end{array}$ & Difference \\
\hline MAX & 1.770 & 1.580 & 0.190 \\
MIN & 0.070 & 0.019 & 0.051 \\
MEAN & 0.762 & 0.367 & 0.394 \\
VAR & 0.2577 & 0.1149 & 0.143 \\
\hline
\end{tabular}

Note: Sample size : 45

\section{Table 5: Statistical results (tax rate $=\mathbf{2 8} \%$ )}

\begin{tabular}{cccc} 
Statistic results & Equity beta & Asset beta (assume debt beta = 0) & Difference \\
\hline MAX & 1.770 & 1.583 & 0.187 \\
MIN & 0.075 & 0.020 & 0.055 \\
MEAN & 0.769 & 0.370 & 0.398 \\
VAR & 0.2546 & 0.1148 & 0.140 \\
\hline
\end{tabular}

Note: Sample size : 45

Table 6: Statistical results (tax rate $=\mathbf{2 0} \%$ )

\begin{tabular}{cccc} 
Statistic results & Equity beta & Asset beta (assume debt beta = 0) & Difference \\
\hline MAX & 1.770 & 1.575 & 0.195 \\
MIN & 0.062 & 0.016 & 0.046 \\
MEAN & 0.750 & 0.362 & 0.388 \\
VAR & 0.2624 & 0.1151 & 0.147 \\
\hline
\end{tabular}

Note: Sample size : 45

Based on the above results, we find out:

Equity beta mean values in all 3 scenarios are low $(<0,8)$ and asset beta mean values are also small $(<0,4)$ although max equity beta values in some cases might be higher than $(>) 1$. In the case of current tax rate of $25 \%$, equity beta value fluctuates in an acceptable range from 0.07 ( $\mathrm{min}$ ) up to 1.77 (max) and asset beta fluctuates from 0,019 ( $\min$ ) up to 1.58 (max). If corporate tax rate increases to $28 \%$, equity beta changes from 0.075 to 1.77 and asset beta move in a range from 0.02 to 1.583 . When tax rate decreases down to $20 \%$, equity beta value changes from 0.062 to 1.77 and asset beta fluctuates in a range from 0.016 to 1.575 .

Beside, Exhibit 6 informs us that in the case $28 \%$ tax rate, average equity beta value of 45 listed firms increases to 0.007 while average asset beta value of these 45 firms increase slightly up to 0.003 . Then, when tax rate reduces to $20 \%$, average equity beta value of 45 listed firms goes down to -0.01 and average asset beta value of 45 firms down to -0.005 .

The below chart 1 shows us : when tax rate decreases down to $20 \%$, average equity and asset beta values increase slightly ( 0.750 and 0.362 ) compared to those at the initial rate of $25 \%$ (0.762 and 0.367), which shows opposite movement compared to the market index. At the same time, when tax rate increases up to $28 \%$, average equity beta decreases slightly whereas average asset beta value remains unchanged (to 0.769 and 0.370 ). However, the fluctuation of equity beta value $(0.262)$ in the case of $20 \%$ tax rate is higher than (>) the results in the rest 2 tax rate cases. 
Chart 1: Comparing statistical results of three (3) scenarios of changing tax rate (2007-2009)

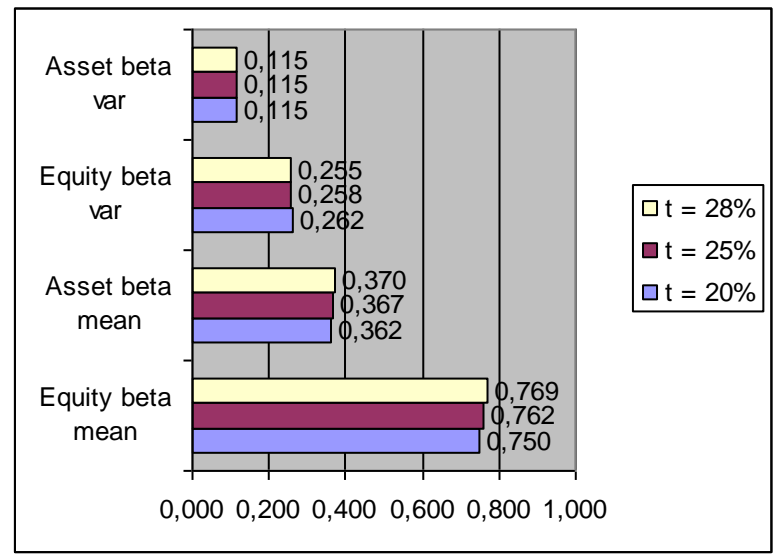

Chart 2 - Comparing statistical results of three (3) scenarios of changing tax rate (20072011)

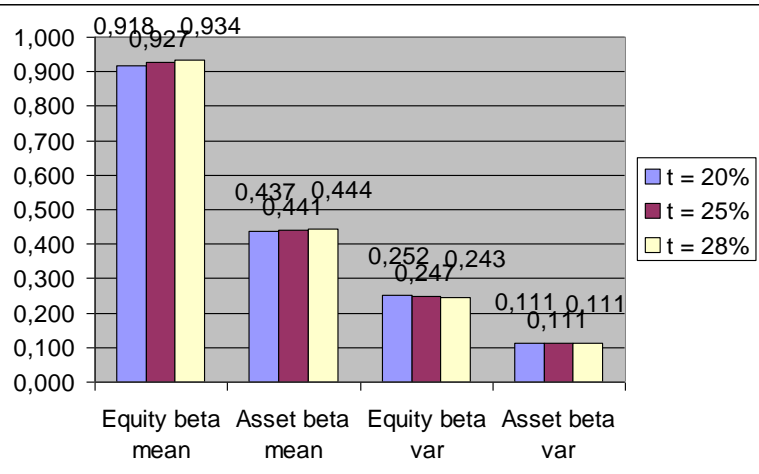

\section{Risk analysis}

On the one hand, in the case of decreasing tax rate, (20\%), the market and companies can receive more benefits such as generating more jobs, output and compensation, but the government budget can have deficit and the government has to cut expenditures. Hence, changes in tax rates can have both positive and negative impacts on the local market.

On the other hand, in the case of increasing tax rate (28\%), the government will have budget to finance public expenditures but the income tax burden could reduce both demand and supply, as well as the output, jobs and compensation.

\section{Conclusion and Policy suggestion}

In summary, the government has to consider the impacts on the mobility of capital in the markets when it changes the tax policy or tax rates. Beside, it continues to increase the effectiveness of building the legal system and regulation and macro policies supporting the plan of developing real estate market. The Ministry of Finance Continue to increase the effectiveness of fiscal policies and tax policies which are needed to combine with other macro policies at the same time, although we could note that in this study when tax rate is going to increase up to $28 \%$, the risk level does not increase so much, compared to the case it is going to decrease down to $20 \%$. And the risk dispersion during 2007-2009 (asset beta var of 0.115) is higher than that during 2007-2011 $(0.111)$ in case tax $25 \%$.

The State Bank of Viet Nam continues to increase the effectiveness of capital providing channels for real estate companies. Furthermore, the entire efforts among many different government bodies need to be coordinated.

Finally, this paper suggests implications for further research and policy suggestion for the Viet Nam government and relevant organizations, economists and investors from current market conditions. 


\section{Appendix A. Supplementary material}

Supplementary data associated with this article can be found, in the online version, at https://doi.org/10.14254/jems.2018.3-2.3

\section{Funding}

The authors received no direct funding for this research.

\section{Citation information}

Huy, Dinh T. N. (2018). Tax rates effects on the risk level of listed Viet Nam real estate firms during global economic crisis 2007-2009. Economics, Management and Sustainability, 3(2), 34-41. doi:10.14254/jems.2018.3-2.3

\section{References}

ADB and Viet Nam Fact Sheet. (2010).

Al Mamun, M. A. (2013). Performance evaluation of prime bank limited in terms of capital adequacy. Global Journal of Management and Business Research, 13(9), 26-29.

Baker, H. K., Singleton, J. C., \& Veit, E. T. (2011). Survey research in corporate finance: bridging the gap between theory and practice. Oxford University Press.

Beenish, A. \& Moazzam, J. (2013). A Test of Fama and French Three Factor Model in Pakistan Equity Market. Global Journal of Management and Business Research, 13(7), 24-28.

Flifel, K. (2012). Financial Markets between Efficiency and Persistence: Empirical Evidence on Daily Data. Asian Journal of Finance \& Accounting, 4(2), 379-400.

Huy, Dinh T.N. (2013). Beta of Viet Nam Listed Computer and Electrical Company Groups During and After The Financial Crisis 2007-2011. Asian Journal of Finance \& Accounting, 5(1), 127 139.

Huy, Dinh T.N. (2013). Estimating beta of Viet Nam listed public utilities, natural gas and oil company groups during and after the financial crisis 2007-2011. Economic and Business Review, 15(1), 57-71.

Ovat, O. O. (2013). Liquidity Constraints and Entrepreneurial Financing in Nigeria: The Fate of Fresh Graduate Entrepreneurs. Global Journal of Management and Business Research, 13(9), 49-57.

Raj, Bhavana., \& Sindhu, Dr. (2013). Skill Level in Risk Management: Training in Credit Risk - A Comparative Study of Indian Banks and Foreign Banks. Global Journal of Management and Business Research, 13, 7, 56-62.

Rehman, S. S. F. U. (2013). Relationship between financial leverage and financial performance: Empirical evidence of listed sugar companies of Pakistan. Global Journal of Management and Business Research, 13(8), 45-53. 


\section{Appendix B}

\section{Exhibit}

\begin{tabular}{cccc} 
Exhibit 1: Interest rates in banking industry during crisis & \\
Year & $\begin{array}{c}\text { Borrowing Interest } \\
\text { rates }\end{array}$ & Deposit Rates & Note \\
\hline 2011 & $18 \%-22 \%$ & $13 \%-14 \%$ & Approximately \\
2010 & $19 \%-20 \%$ & $13 \%-14 \%$ & $\begin{array}{c}\text { (2007: required reserves ratio at SBV is } \\
\text { changed from } 5 \% \text { to } 10 \%)\end{array}$ \\
2009 & $9 \%-12 \%$ & $9 \%-10 \%$ & $(2009:$ special supporting interest rate is \\
2008 & $19 \%-21 \%$ & $15 \%-16,5 \%$ & $4 \%)$ \\
2007 & $12 \%-15 \%$ & $9 \%-11 \%$ & \\
\hline
\end{tabular}

(Source: Viet Nam commercial banks)

\section{Exhibit 2: Basic interest rate changes in Viet Nam}

\begin{tabular}{ccc} 
Year & Basic rate & Note \\
\hline 2011 & $9 \%$ & \\
2010 & $8 \%$ & \\
2009 & $7 \%$ & \\
2008 & $8.75 \%-14 \%$ & \\
2007 & $8.25 \%$ & \\
2006 & $8.25 \%$ & \\
2005 & $7.8 \%$ & \\
2004 & $7.5 \%$ & Approximately, fluctuated \\
2003 & $7.5 \%$ & \\
2002 & $7.44 \%$ & \\
2001 & $7.2 \%-8.7 \%$ & \\
2000 & $9 \%$ & \\
\hline
\end{tabular}

(Source: State Bank of Viet Nam and Viet Nam economy)

\begin{tabular}{cccc} 
Exhibit 3: Inflation, GDP growth and macroeconomics factors & \\
Year & Inflation & GDP & USD/VND rate \\
\hline 2011 & $18 \%$ & $5.89 \%$ & 20.670 \\
2010 & $11.75 \%$ (Estimated & $6.5 \%$ & 19.495 \\
2009 & at Dec 2010) & (expected) & 17.000 \\
2008 & $6.88 \%$ & $5.2 \%$ & 17.700 \\
2007 & $22 \%$ & $6.23 \%$ & 16.132 \\
2006 & $12.63 \%$ & $8.44 \%$ & \\
2005 & $6.6 \%$ & $8.17 \%$ & \\
\hline
\end{tabular}

Note

approximately

(Source: Viet Nam commercial banks and economic statistical bureau) 


\section{Exhibit 4: GDP growth Việt Nam 2006-2010 (source: Bureau Statistic)}

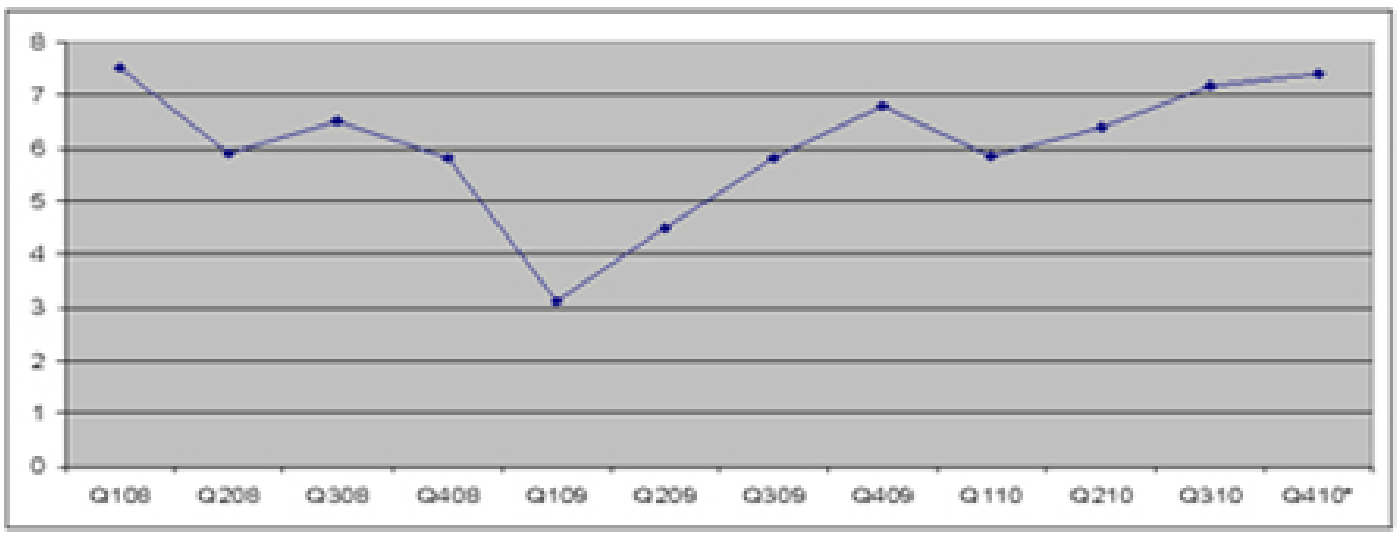

Exhibit 5: Risk and financial leverage of 9 listed banking firms on VN stock exchange period 2007-2011

\begin{tabular}{ccccc}
$\begin{array}{c}\text { Order } \\
\text { No. }\end{array}$ & $\begin{array}{c}\text { Company stock } \\
\text { code }\end{array}$ & $\begin{array}{c}\text { Equity } \\
\text { beta }\end{array}$ & $\begin{array}{c}\text { Asset beta (assume debt beta }= \\
\text { 0) }\end{array}$ & $\begin{array}{c}\text { Financial } \\
\text { leverage }\end{array}$ \\
\hline 1 & ACB & 0.7874 & 0.0378 & $95.2 \%$ \\
2 & CTG & 0.5540 & 0.0312 & $94.4 \%$ \\
3 & EIB & 0.3847 & 0.0365 & $90.5 \%$ \\
4 & HBB & 0.1335 & 0.0138 & $89.7 \%$ \\
5 & MBB & 0.0722 & 0.0054 & $92.5 \%$ \\
6 & NVB & 0.0211 & 0.0026 & $87.7 \%$ \\
7 & SHB & 1.0038 & 0.0824 & $91.8 \%$ \\
8 & STB & 0.7395 & 0.0721 & $90.3 \%$ \\
9 & VCB & 0.4083 & 0.0299 & $92.7 \%$ \\
\hline
\end{tabular}


Exhibit 6: Increase/decrease risk level of listed real estate firms under changing scenarios of

tax rates : 25\%, 28\%, 20\% period $2007-2009$

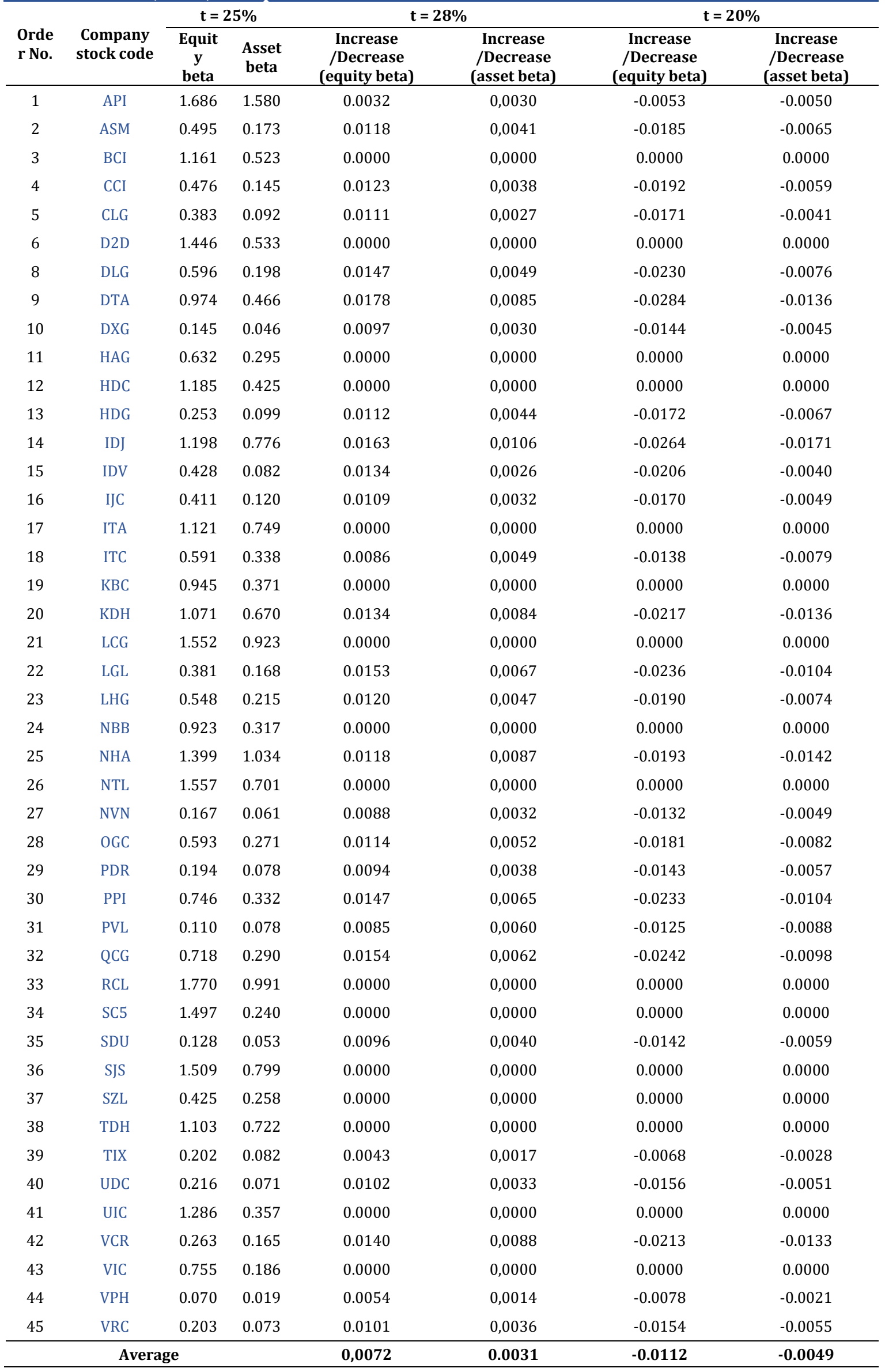




\section{Exhibit 7- VNI Index and other stock market index during crisis 2006-2010}
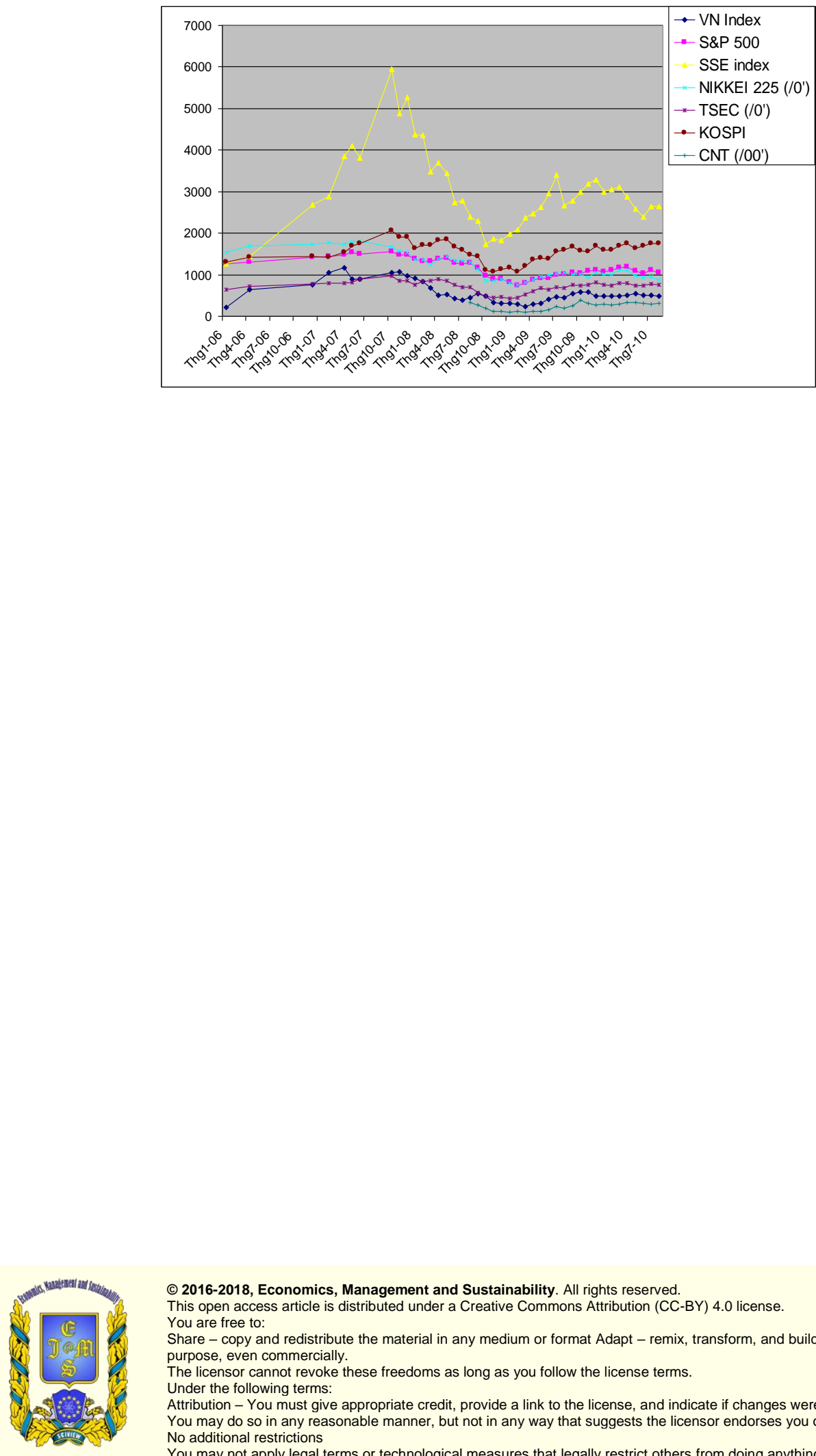

() 2016-2018, Economics, Management and Sustainability. All rights reserved.

This open access article is distributed under a Creative Commons Attribution (CC-BY) 4.0 license.

You are free to:

Share - copy and redistribute the material in any medium or format Adapt - remix, transform, and build upon the material for any

purpose, even commercially.

The licensor cannot revoke these freedoms as long as you follow the license terms.

Under the following terms:

Attribution - You must give appropriate credit, provide a link to the license, and indicate if changes were made.

You may do so in any reasonable manner, but not in any way that suggests the licensor endorses you or your use.

No additional restrictions

You may not apply legal terms or technological measures that legally restrict others from doing anything the license permits.

Economics, Management and Sustainability (ISSN: 2520-6303) is published by Scientific Publishing House "CSR",

Poland, EU and Scientific Publishing House "SciView", Poland

Publishing with JEMS ensures:

- Immediate, universal access to your article on publication

- High visibility and discoverability via the JEMS website

- Rapid publication

- Guaranteed legacy preservation of your article

- Discounts and waivers for authors in developing regions

Submit your manuscript to a JEMS at http://jems.sciview.net or submit.jems@sciview.net

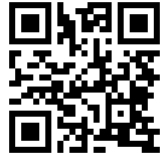

